\title{
Opportunities and Challenges: Human Papillomavirus and Cancer
}

\author{
Presented by Christina S. Chu, MD, and David G. Pfister, MD
}

\begin{abstract}
Mucosal exposure to human papillomavirus (HPV) can lead to anogenital and head and neck (H\&N) cancer. Vaccination at a young age can be almost $100 \%$ effective in preventing HPV infection with the viral subtypes in both men and women, at least for disease in the anogenital tract. Therapeutic strategies targeting HPV in cervical dysplasia and cancer are showing promise as well in regressing dysplasia and controlling disease. That HPV-positive H\&N cancer is a different disease from HPV-negative disease, with different molecular and clinical features and prognosis, is becoming better appreciated. At this time, however, the NCCN Guidelines for H\&N Cancers do not distinguish between the types. This is expected to change.
\end{abstract}

J Natl Compr Canc Netw 2017;15(5.5):726-729

Human papillomavirus (HPV) is a double-stranded DNA virus of $>100$ subtypes. Through close mucosal contact, high-risk subtypes can facilitate the development of epithelial cancers and can influence tumor activity and disease prognosis. The good news is that HPV infection can be prevented and the related cancers can be targeted therapeutically, as described by 2 experts on HPV-related disease at the recent NCCN 22nd Annual Conference.

Christina S. Chu, MD, Associate Professor of Gynecologic Oncology, Fox Chase Cancer Center, discussed HPV-related cervical cancer, and David G. Pfister, MD, Chief, Head and Neck Oncology Service, Memorial Sloan Kettering Cancer Center, and Professor of Medicine at Weill Cornell Medical College, described the evolving role of HPV in head and neck $(\mathrm{H} \& \mathrm{~N})$ cancer.

Presented by Christina S. Chu, MD, Gynecologic Oncology, Fox Chase Cancer Center, Philadelphia, Pennsylvania; and David G. Pfister, MD, Head and Neck Oncology Service, Memorial Sloan Kettering Cancer Center, and Weill Cornell Medical College, New York, New York.

Dr. Chu has disclosed that she has no financial interests, arrangements, affiliations, or commercial interests with the manufacturers of any products discussed in this article or their competitors. Dr. Pfister has disclosed that he has received consulting fees from Boehringer Ingelheim $\mathrm{GmbH}$ and grant or research support from AstraZeneca Pharmaceuticals LP, Bayer HealthCare, Eli Lilly and Company, Exelixis Inc., Genentech, Inc., GlaxoSmithKline, MedImmune Inc., Merck \& Co., Inc., and Novartis Pharmaceuticals Corporation.

Correspondence: Christina S. Chu, MD, Fox Chase Cancer Center, 333 Cottman Avenue, Philadelphia, PA 19111.

E-mail: Christina.Chu@fccc.edu

David G. Pfister, MD, Memorial Sloan Kettering Cancer Center, 1275

York Avenue, Box 188, New York, NY 10065. E-mail: pfisterd@mskcc.org

\section{Cervical and Vaginal Cancers}

Approximately $40 \mathrm{HPV}$ subtypes are relevant to disease in the anogenital tract. Subtypes 16 and 18 are responsible for approximately $70 \%$ of cervical cancer cases, and subtypes 6 and 11 cause $90 \%$ of genital warts. ${ }^{1}$

"For the most part, we consider HPV infection a ubiquitous asymptomatic disease," Dr. Chu said. The lifetime risk for sexually active men and women exceeds $50 \% .{ }^{1}$ By age 50 years, approximately $80 \%$ of women have acquired genital HPV infection, ${ }^{1}$ and for women aged $\leq 25$ years, the prevalence rate approaches $50 \%$. Almost three-quarters of new HPV infections occur in persons aged 15 to 24 years. $^{3}$

Although the immune system often clears the virus without sequelae, HPV infection can persist and evolve into dysplasia, high-grade dysplasia, and ultimately cancer. Some of these mild dysplastic changes are never fated to become cancer, but "we can't tell the difference between a truly 'precancerous' lesion and one that is not," Dr. Chu said. "These days, we focus on high-risk subtypes of infection and high-grade dysplasia."

The premalignant condition vulvar intraepithelial neoplasia (VIN) is also HPV-related and can progress to vulvar squamous cell cancer. HPV infection is responsible for $40 \%$ of these cases, which tend to occur in younger women; older women are likely to have HPV-negative disease associated with vulvar dystrophies. Depending on severity, treatment involves wide local excision, laser ablation, or topical imiquimod. 
"Across the timeline of HPV [anogenital] infection, we can intervene in a few places: with primary prevention through vaccination, with targeting of HPV once precancerous lesions have developed, and with anti-HPV strategies to treat invasive cancer," Dr. Chu offered.

\section{Preventive Vaccination Nearly $100 \%$ Effective}

Almost complete prevention of HPV infection by the targeted subtypes is possible with the 4-valent vaccine (which covers subtypes $6,11,16$, and 18 ) and the 9-valent vaccine (which covers $6,11,16$, $18,31,33,45,52$, and 58). A systematic review of 10 years of "real-world" experience with the 4-valent vaccine concluded that it prevents up to $90 \%$ of HPV 6/11/16/18 infections and $85 \%$ of highgrade cervical dysplasia. ${ }^{4}$ The 9 -valent product was subsequently approved based on equal efficacy in a comparative trial (Figure 1). ${ }^{5}$

Vaccination should be administered before individuals (both genders) become sexually active; otherwise its efficacy wanes. It should be given through the age of 26 years to women, men who have sex with men, transgender adults, and persons with immunocompromising conditions, and through the age

\section{A}

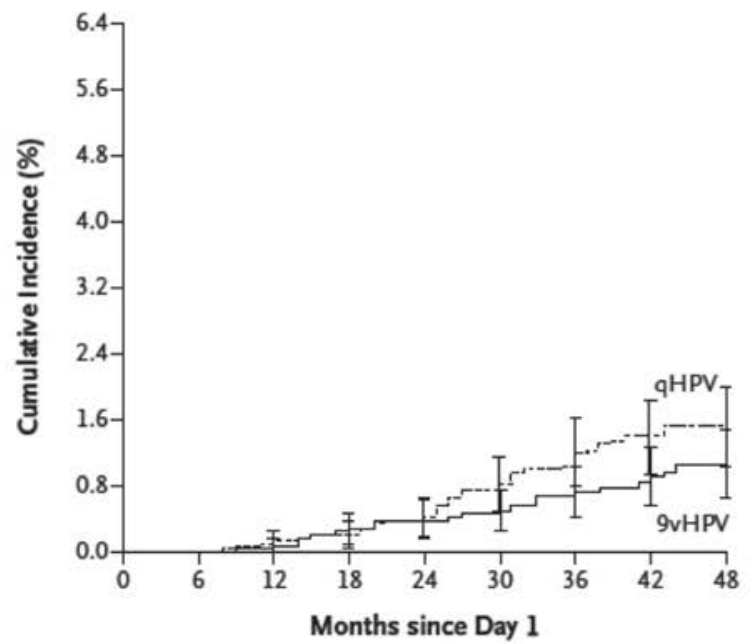

\section{No. at Risk}

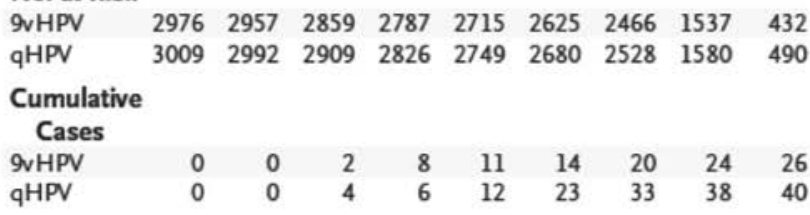

of 21 years to boys and men. "Unfortunately, only $60 \%$ of teenage girls actually initiate the vaccine," Dr. Chu noted. "The good news is that up to $50 \%$ of boys are now being vaccinated."

\section{Immunotherapeutic Strategies}

Immunotherapeutic approaches for high-grade dysplasia and cervical cancer are moving forward, incorporating bacterial- and viral-based vectors and peptide, protein, DNA, and dendritic cell vaccines.

VGX-3100 is the first vaccine to result in regression of precancerous lesions. In a phase II study of 167 women with HPV infection with high-grade dysplasia, regression was observed in $49.5 \%$ of women in the vaccine arm versus $20 \%$ of women in the placebo arm. ${ }^{6}$ Another immunotherapeutic agent is the Listeria-based fusion product axalimogene filolisbac (ADXS11-001). This led to an impressive 12-month overall survival rate of $38 \%$ in a study of 50 patients with recurrent or metastatic cervical cancer. ${ }^{7}$ The phase III AIM2CERV trial will randomize patients with locally advanced cervical cancer in remission after chemoradiotherapy to ADXS11-001 or placebo, with the end point of overall survival.

B

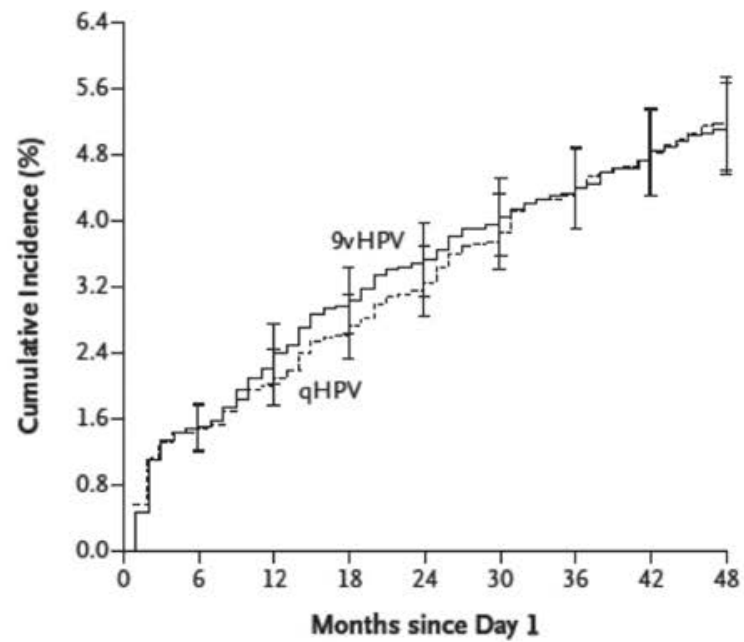

No. at Risk

$\begin{array}{llllllllll}\text { 9vHPV } & 6882 & 6710 & 6449 & 6253 & 6079 & 5853 & 5483 & 3487 & 964\end{array}$ $\begin{array}{llllllllll}\text { qHPV } & 6871 & 6700 & 6475 & 6286 & 6093 & 5899 & 5552 & 3532 & 1039\end{array}$

Cumulative

Cases

$\begin{array}{llllllllll}9 \mathrm{vHPV} & 0 & 103 & 163 & 205 & 237 & 270 & 290 & 314 & 322\end{array}$ $\begin{array}{llllllllll}\text { qHPV } & 0 & 101 & 143 & 184 & 219 & 257 & 289 & 312 & 323\end{array}$

Figure 1. Results for the 9-valent human papilloma virus (HPV) vaccine irrespective of subtype among (A) participants not HPV-infected on day 1 , and $(B)$ the modified intention-to-treat population.

From Joura EA, Giuliano AR, Iversen O-E, et al. A 9-valent HPV vaccine against infection and intraepithelial neoplasia in women. N Engl J Med 2015;372:711-723. Reprinted with permission from Massachusetts Medical Society. @2015. 
Dr. Chu closed by observing that "even in our own country," tremendous disparities in cancer diagnosis and care still exist. "We still have work to do," she said.

\section{HPV-Positive H\&N Cancer}

"Although H\&N cancer is strongly associated with tobacco and alcohol use, prior HPV infection is increasingly appreciated as a risk factor as well," said Dr. Pfister.

In fact, although HPV-positive cancers are on the rise, HPV-negative cases-those most often related to tobacco and alcohol—are declining. HPVrelated cancers tend to be diagnosed in younger persons (mostly white men) and to be associated with increased numbers of sexual partners and certain sexual practices.

"These are really 2 different diseases," Dr. Pfister stated. For HPV-positive cancer, the oropharynx and tonsils are the most common primary sites and HPV16 is the most common subtype. HPV-positive and HPV-negative tumors are molecularly different and can have distinct pathologic features and clinical presentations. With both types, distant metastases are uncommon at presentation.

Although any cancer can have a "locoregional character," one often finds a small primary but bulky neck nodes in patients with HPV-positive disease. At times, primaries may even be occult, although most "turn out to be buried in the oropharynx," Dr. Pfister said. Finding HPV positivity in the neck node can help focus the workup and local treatment, and thus potentially reduce morbidity.

Unlike HPV infection of the cervix, there is no formal preventive or screening strategy for HPV-related $\mathrm{H} \& \mathrm{~N}$ cancer. That said, it is prudent to counsel patients on how the virus is spread, and it is expected that HPV vaccination should ultimately decrease the incidence of HPV-related H\&N cancers. Also, direct inspection and palpation of the oral cavity and oropharynx during dental examinations may reveal some of these cancers.

\section{Prognosis and Treatment}

"HPV-related H\&N cancer is more responsive to initial treatment and has a better prognosis than HPV-negative disease," Dr. Pfister said. ${ }^{8}$ Even with recurrent or metastatic disease, patients with HPV-related cancer live longer, accord- ing to a retrospective analysis of RTOG 0129/0522 that found median survival to be 2.6 versus 0.8 years, respectively $(P<.001) .{ }^{9}$

Despite the better prognosis, HPV-positive cancers are managed the same as HPV-negative tumors. For early-stage disease, single-modality therapy (surgery or radiotherapy) is recommended. For locoregionally advanced disease, a combined-modality approach is taken. If the tumor is resectable, surgery or chemoradiotherapy with definitive intent is recommended; the addition of chemotherapy has become part of the standard of care for many patients in this setting. For unresectable tumors, patients proceed directly to chemoradiotherapy. For recurrent or metastatic disease, selected patients receive surgery, repeat irradiation, or chemoirradiation, with the hope of more durable disease control. Chemotherapy and other systemic therapy may improve survival, but by itself is not a curative modality; it is primarily administered with palliative intent.

"In the NCCN Guidelines, we don't yet have an HPV-positive page and HPV-negative page, though I expect that in the future this will evolve," Dr. Pfister said. "You do see a hint that we are starting to look at this-the workup for oropharynx cancer differs from that of other primary sites in that HPV testing is recommended. This doesn't necessarily change the treatment, but it changes the prognosis and how you counsel patients."

Part of the problem, Dr. Pfister noted, is that current standards of care typically stem from clinical trials that did not check HPV status. For instance, based on data from seminal studies of patients with poor risk pathologic factors after primary surgery, the

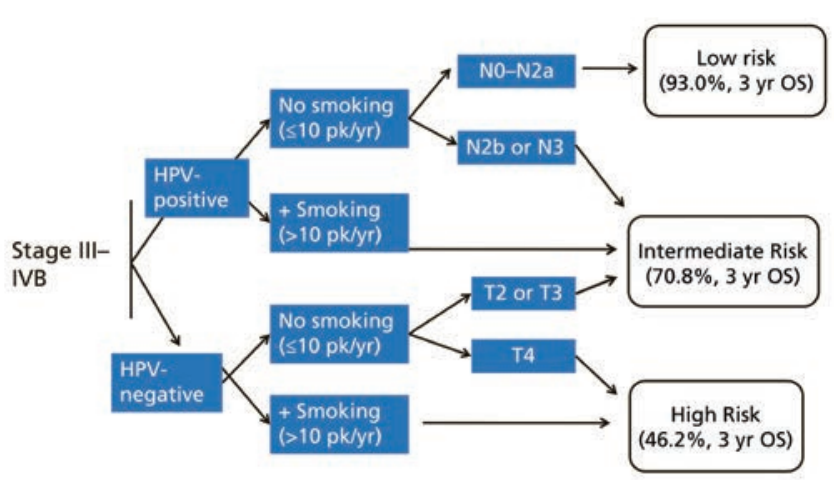

Figure 2. Prognosis of oropharynx cancer.

Abbreviations: HPV, human papilloma virus; OS, overall survival; pk, pack.

Adapted from Ang et al. Human papillomavirus and survival of patients with oropharyngeal cancer. N Engl J Med 2010;363:24-35. 
NCCN Guidelines have listed concurrent cisplatin plus radiotherapy as a category 1 recommendation in the postoperative setting for patients with extracapsular nodal spread or positive margins. However, recent institutional data suggest that extracapsular spread may not carry the same adverse prognosis for HPV-positive patients. The benefits of cisplatin plus radiotherapy may be more controversial in this HPV subgroup. The NCCN H\&N Cancers Panel has therefore retained systemic therapy with radiation as a category 1 recommendation for primary sites ex- cept for the oropharynx, where it is now a category $2 \mathrm{~A}$ recommendation.

\section{Future Strategies}

In the future, staging may become HPV status-specific and the NCCN Guidelines algorithms may change. Researchers are also asking whether HPVpositive patients can be treated less aggressively and are evaluating means of less-aggressive treatment that will not compromise survival. Finally, novel agents being evaluated for $\mathrm{H} \& \mathrm{~N}$ cancer may have different activity for HPV-positive versus HPVnegative disease; studies are ongoing.

\section{References}

1. HPV-Associated Cancer Statistics. Centers for Disease Control and Prevention Web site. Available at: https://www.cdc.gov/cancer/hpv/ statistics/index.htm. Accessed March 28, 2017.

2. Bauer HM, Ting Y, Greer CE, et al. Genital human papillomavirus infection in female university students as determined by a PCR-based method. JAMA 1991;265:472-477.

3. Weinstock H, Berman S, Cates W Jr. Sexually transmitted diseases among American youth: incidence and prevalence estimates, 2000. Perspect Sex Reprod Health 2004;36:6-10.

4. Garland SM, Kjaer SK, Munoz N, et al. Impact and effectiveness of the quadrivalent human papillomavirus vaccine: a systematic review of 10 years of real-world experience. Clin Infect Dis 2016;63:519-527.

5. Joura EA, Giuliano AR, Iversen OE, et al. A 9-valent HPV vaccine against infection and intraepithelial neoplasia in women. N Engl J Med 2015;372:711-723.

6. Trimble CL, Morrow MP, Kraynyak KA, et al. Safety, efficacy, and immunogenicity of VGX-3100, a therapeutic synthetic DNA vaccine targeting human papillomavirus 16 and $18 \mathrm{E} 6$ and E7 proteins for cervical intraepithelial neoplasia 2/3: a randomised, double-blind, placebocontrolled phase 2b trial. Lancet 2015;386:2078-2088.

7. Huh W. Brady WE, Dizon DS, et al. A prospective phase II trial of the listeria-based human papillomavirus immunotherapy axalimogene filolisbac in second- and third-line metastatic cervical cancer: a NRG oncology group trial [abstract]. Presented at the 48th Annual Meeting of the Society of Gynecologic Oncology; March 12-15, 2017; National Harbor, Maryland. Abstract LBA3.

8. Ang KK, Harris J, Wheeler R, et al. Human papillomavirus and survival of patients with oropharyngeal cancer. N Engl J Med 2010;363:24-35.

9. Fakhry C, Zhang Q, Nguyen-Tan PF, et al. Human papillomavirus and overall survival after progression of oropharyngeal squamous cell carcinoma. J Clin Oncol 2014;32:3365-3373. 